University of Nebraska - Lincoln

DigitalCommons@University of Nebraska - Lincoln

\title{
4-1-1981
}

\section{Rare-earth-rich metallic glasses. II. Magnetic viscosity}

George C. Hadjipanayis

University of Nebraska-Lincoln, hadji@udel.edu

David J. Sellmyer

University of Nebraska-Lincoln, dsellmyer@unl.edu

Follow this and additional works at: https://digitalcommons.unl.edu/physicssellmyer

Part of the Physics Commons

Hadjipanayis, George C. and Sellmyer, David J., "Rare-earth-rich metallic glasses. II. Magnetic viscosity" (1981). David Sellmyer Publications. 164.

https://digitalcommons.unl.edu/physicssellmyer/164

This Article is brought to you for free and open access by the Research Papers in Physics and Astronomy at DigitalCommons@University of Nebraska - Lincoln. It has been accepted for inclusion in David Sellmyer Publications by an authorized administrator of DigitalCommons@University of Nebraska - Lincoln. 


\title{
Rare-earth-rich metallic glasses. II. Magnetic viscosity
}

\author{
G. Hadjipanayis and D. J. Sellmyer \\ Behlen Laboratory of Physics, University of Nebraska, Lincoln, Nebraska 68588
}

(Received 7 July 1980)

\begin{abstract}
Magnetic viscosity measurements on amorphous $\mathrm{Tb}_{75} \mathrm{Au}_{25}$ and $\left(\mathrm{Er}_{65} \mathrm{Fe}_{35}\right)_{90} \mathrm{~B}_{10}$ alloys are presented in the temperature range of 1.3 to $30 \mathrm{~K}$. The magnetization in a steady magnetic field is found to follow a logarithmic time dependence for times up to $400 \mathrm{~s}$ suggesting a spectrum of activation energies. The magnetic viscosity parameters were used to investigate the temperature dependence of the observed large coercive fields and to study intrinsic temperature and thermal activation effects. It is found that the large change of the coercive field at very low temperaures is mainly due to thermal activation of domains over the energy barriers.
\end{abstract}

\section{INTRODUCTION}

If the magnetic field applied to a ferromagnetic material is suddenly increased or decreased, the magnetization does not acquire its equilibrium value instantaneously but continues to change as shown in Fig. 1. For the majority of materials the magnetization $M$ is found to vary with the time $t$ according to

$$
M=\text { const }+S \ln t,
$$

where $S$ is the magnetic aftereffect coefficient. The magnitude and the change of the magnetization is too large to be accounted for by eddy currents and is due to thermal activation of domains or domain walls over the free energy barriers.
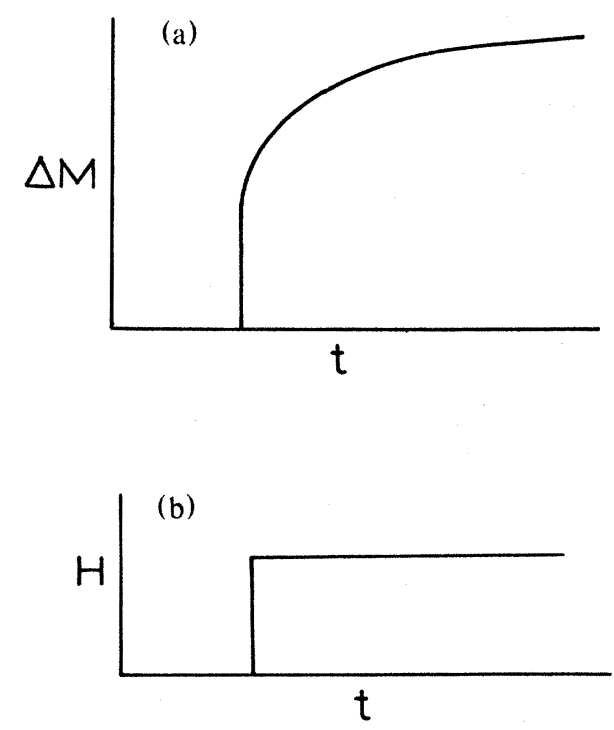

FIG. 1. Variation of magnetization with time (a) after the instantaneous application of a magnetic field (b).
Street and Wooley ${ }^{1}$ were the first to derive the logarithmic time dependence of the magnetization. They showed that $S$ is given by

$$
S=k_{B} T \chi_{\mathrm{ir}} / \frac{d E}{d H},
$$

where $E$ is the activation energy for an energy barrier $H$ the magnetic field, and $\chi_{\text {ir }}$ the irreversible susceptibility $(\partial M / \partial H)$ at the field where $S$ is measured. Gaunt has shown ${ }^{2}$ that in the absence of intrinsic temperature variation of the activation energy $E$, the following relation is obeyed

$$
\left(\frac{\partial E}{\partial H}\right)_{T}=28.5 k_{B} / \frac{d H_{c}}{d T},
$$

where $H_{c}$ is the coercive field. Thus by measuring $S$, $\chi_{\text {ir }}$, and $H_{c}$ over a range of temperatures it is possible to investigate the validity of Eq. (3) and to separate thermal activation and intrinsic temperature contributions to the coercive field $H_{c}$.

Recently Boucher and Barbara ${ }^{3}$ have observed strong magnetic aftereffects in amorphous $\mathbf{T b}-\mathbf{A g}$ alloys prepared by sputtering. However no attempt was made to relate these measurements with the other measured magnetic properties. The work reported here is based on the amorphous rare-earth alloys $\mathrm{Tb}_{75} \mathrm{Au}_{25}$ and $\left(\mathrm{Er}_{65} \mathrm{Fe}_{35}\right)_{90} \mathrm{~B}_{10}$ and was carried out to determine the role of thermal activation in the temperature dependence of the observed high coercive fields. ${ }^{4,5}$

\section{EXPERIMENTAL}

The experimental techniques for sample preparation and for the magnetization measurements have been described elsewhere. ${ }^{4}$ Magnetic viscosity has been measured in the following way: At a given temperature the sample was subjected to fields up to 80

3355 ๑1981 The American Physical Society 

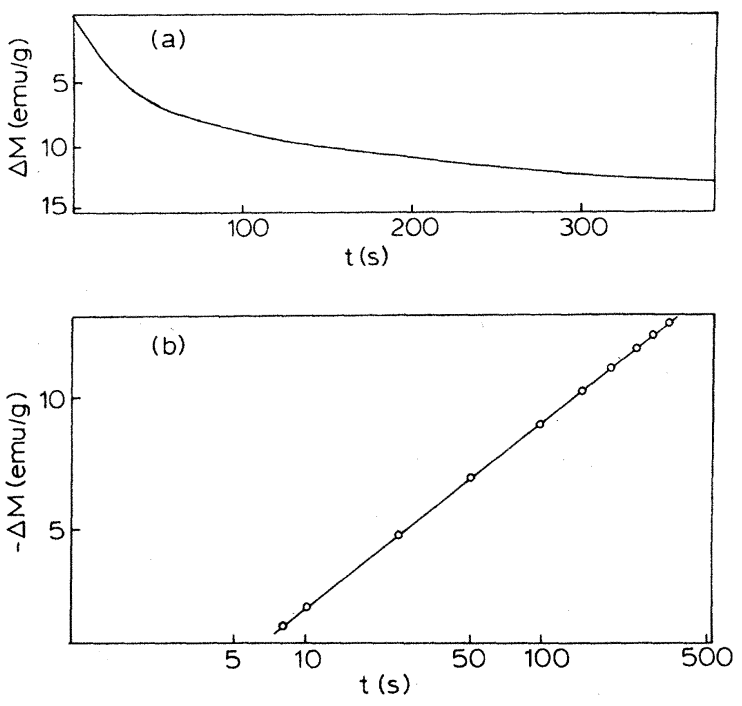

FIG. 2. (a) Output trace from an $x-y$ recorder showing the time dependence of magnetization after the reversed field was increased to a chosen stable value. (b) Change in magnetization as a function of time (time in logarithmic scale).

kOe. The applied field was then reduced to zero slowly with a sweep rate of $60 \mathrm{Oe} / \mathrm{s}$ and then was inverted. The reversed field was increased to a chosen stable value and the subsequent change in magnetization with time was noted by feeding the output signal to an $x-y$ recorder giving an output trace as shown in Fig. 2(a). These traces were examined at different magnetic fields and for different temperatures. The plot of $\Delta M$ (change in magnetization) against ln (time) shows a linear relationship up to $400 \mathrm{~s}$ [Fig. 2 (b)]. The slope of this curve gives the magnetic aftereffect coefficient $S$.

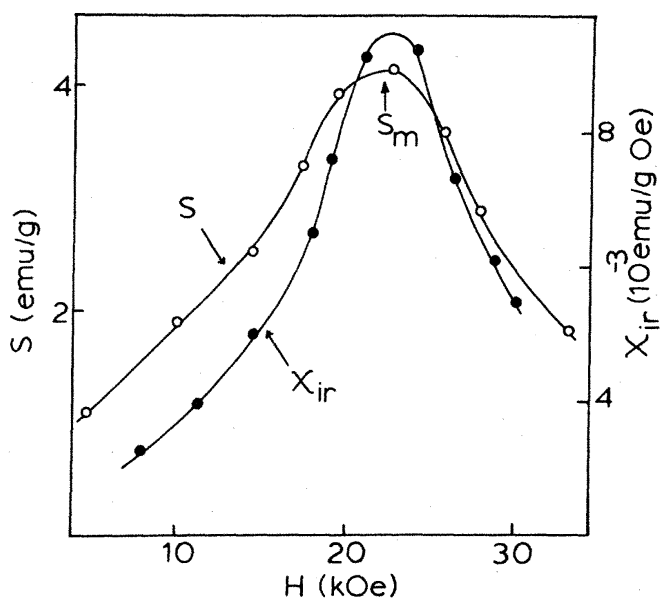

FIG. 3. Aftereffect coefficient $S$ and irreversible susceptibility $\chi_{\text {ir }}$ as a function of reversed field in $\mathrm{Tb}_{75} \mathrm{Au}_{25}$ at $4.2 \mathrm{~K}$.

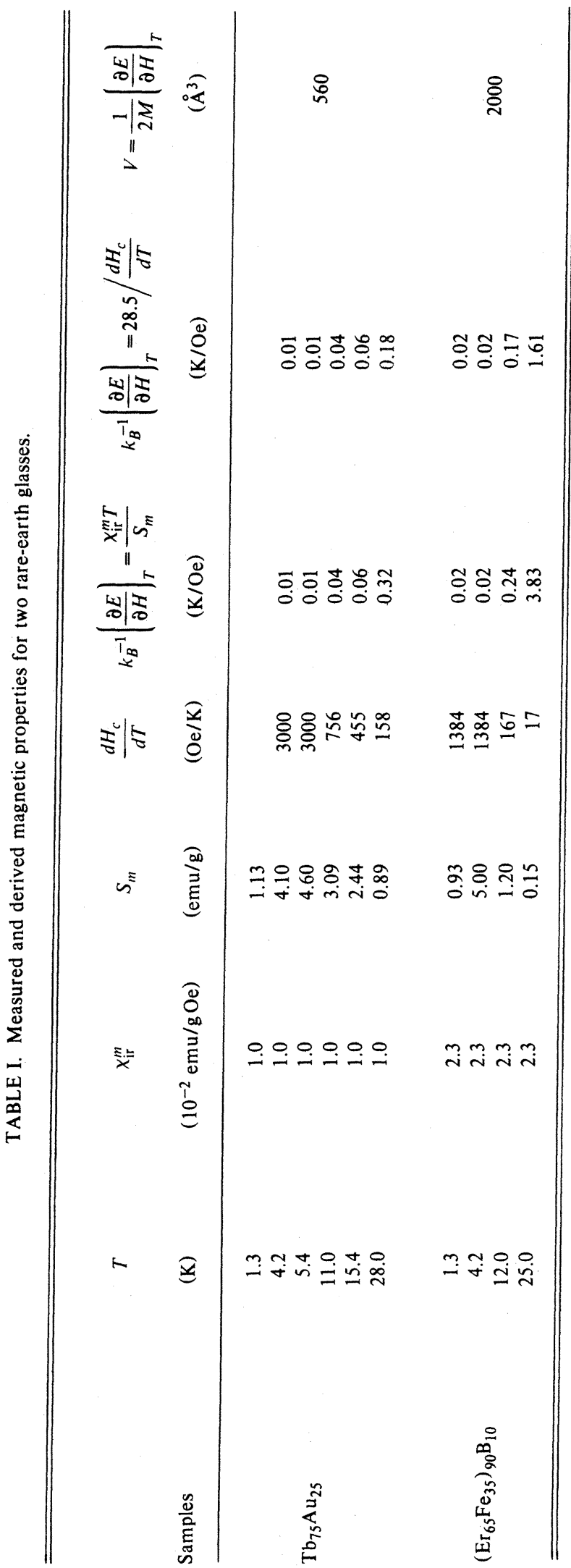




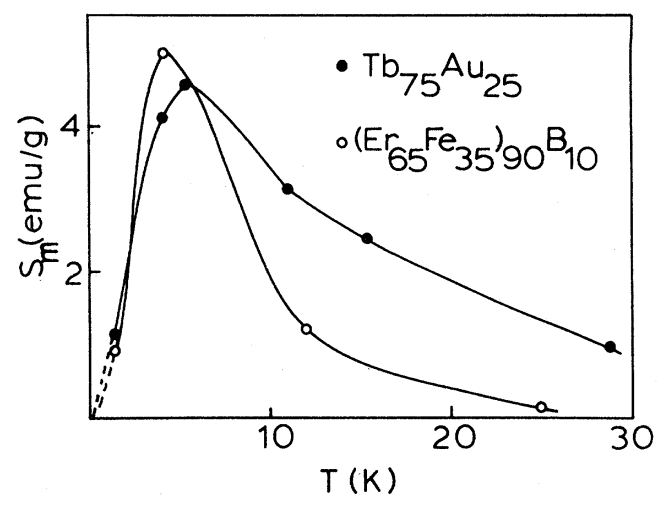

FIG. 4. Temperature dependence of magnetic aftereffect coefficient $S_{m}$.

\section{RESULTS}

The magnetic aftereffect coefficient was found to increase with the reversed field $H$ and peaks around the coercive field $H_{c}$ (Fig. 3 ). This behavior closely resembles the field dependence of the irreversible susceptibility $\chi_{\mathrm{ir}}$ (Fig. 3). The maximum values of $S$ and $\chi_{\mathrm{ir}}, S_{m}$ and $\chi_{\mathrm{ir}}^{m}$, respectively, reflect the behavior

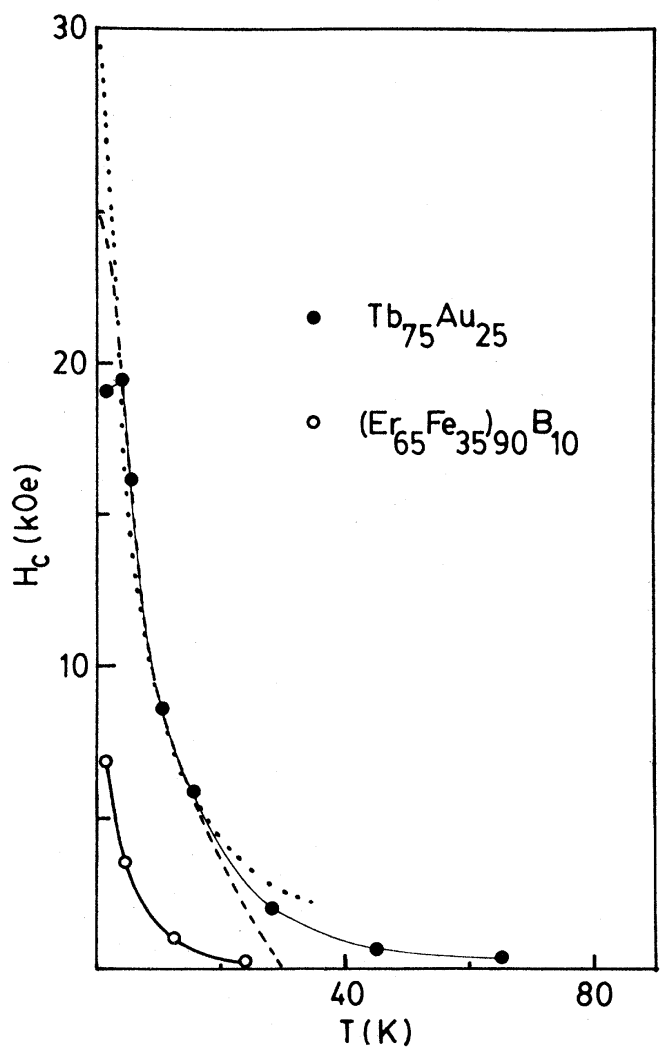

FIG. 5. Temperature dependence of coercive field $H_{c}$ with the theoretical predictions of Gaunt's (dashed-line) and Egami's (dotted-curve) models.

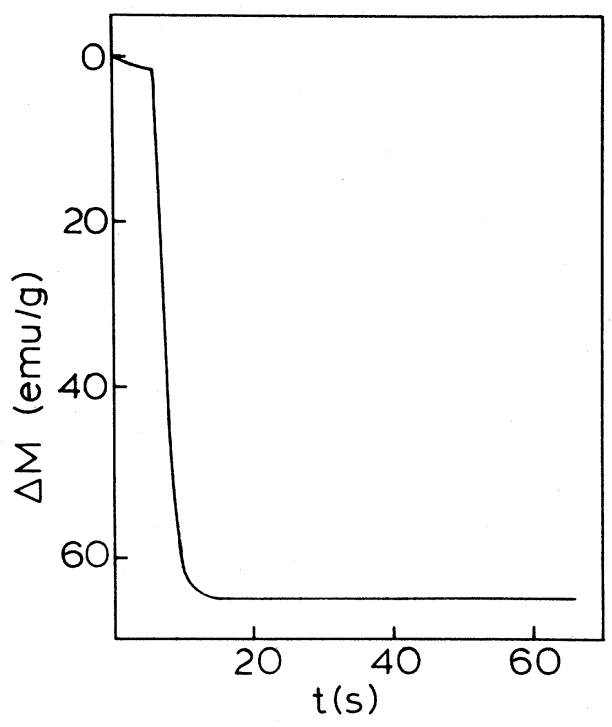

FIG. 6. Magnetization jumps during a viscosity measurement in $\left(\mathrm{Er}_{65} \mathrm{Fe}_{35}\right)_{90} \mathrm{~B}_{10}$ at $1.3 \mathrm{~K}$.

of the most numerous energy barriers and are used for the subsequent analysis of the measurements. Figure 4 shows the temperature variation of $S_{m}$. At absolute zero the coefficient seems to extrapolate to zero as expected since there is no activation. The irreversible susceptibility was found to be temperature independent in the temperature range covered.

Viscosity measurements were made only up to about $30 \mathrm{~K}$ because the coercive fields and magnetization values became'small above that temperature; the ordering temperatures of the $\mathrm{Er}$ and $\mathrm{Tb}$ glasses are 16 and $85 \mathrm{~K}$, respectively. Values of $(\partial E / \partial H)_{T}$ were found using Eq. (2) and they are listed on Table I. The temperature dependence of the coercive fields is shown on Fig. 5.

In $\left(\mathrm{Er}_{65} \mathrm{Fe}_{35}\right)_{90} \mathrm{~B}_{10}$ large magnetization jumps were observed at $1.3 \mathrm{~K}$ (Fig 6). However after the magnetization jump the change in magnetization with time (in a constant field) is almost negligible.

\section{DISCUSSION}

The probability of a domain overcoming an energy barrier of height $E$ is given by the mean waiting time, $\tau$,

$$
\tau^{-1}=C e^{-E / k_{B} T}
$$

where $C$ is a constant of ten taken as $\exp (25) \mathrm{Hz}^{6}$ For a static experiment from 1 to $1000 \mathrm{~s}$, energy barriers whose heights are between 25 and $31.9 k_{B} T$ will contribute to the magnetic aftereffect during this time interval. The In (time) dependence of the magneti- 
zation suggests a spectrum of energy barriers, with an average activation energy of $28.5 k_{B} T$.

In general the activation energy is a function of the temperature and magnetic field. ${ }^{2}$ Thus

$$
\frac{d E}{d T}=\left(\frac{\partial E}{\partial H}\right)_{T} \frac{d H}{d T}+\left(\frac{\partial E}{\partial T}\right)_{H} .
$$

At $S_{m}$ Eq. (5) can be rewritten as

$$
28.5 k_{B}=\left(\frac{\partial E}{\partial H}\right)_{T} \frac{d H_{c}}{d T}+\left(\frac{\partial E}{\partial T}\right)_{H} .
$$

In the absence of intrinsic temperature variation of the activation energy $E,(\partial E / \partial T)_{H}=0$, Eq. (3) can be derived

$$
\left(\frac{\partial E}{\partial H}\right)_{T}=28.5 k_{B} / \frac{d H_{c}}{d T} .
$$

The left-hand side of the above equation is found from magnetic-viscosity measurements using Eq. (2). The right-hand side is calculated from the slope of the temperature dependence of the coercive field (Table I). In $T b_{75} A_{u_{25}}$ this equation is very well satisfied in most of the temperature range covered. Thus the large increase of the coercive field on cooling is mainly due to the increased difficulty of thermal activation over energy barriers at low temperatures. This is consistent with high-field magnetization measurements (Fig. 7) which show that in this temperature range the intrinsic properties, the anisotropy $K$ and the saturation magnetization $M_{0}$, are fairly constant and do not affect the strength of the energy barrier. However in $\left(\mathrm{Er}_{65} \mathrm{Fe}_{35}\right)_{90} \mathrm{~B}_{10}$ the agreement is very good at helium temperatures. At higher temperatures deviations occur suggesting intrinsic temperature contributions to the temperature dependence of the coercivity. This is also consistent with

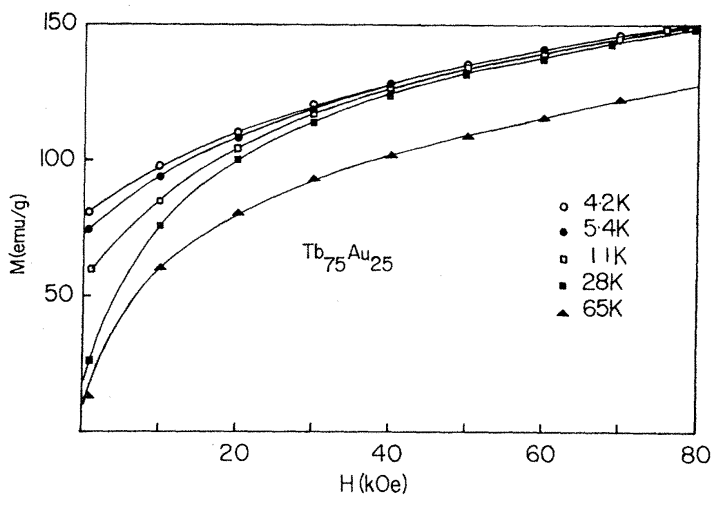

FIG. 7. Magnetization curves for $\mathrm{Tb}_{75} \mathrm{Au}_{25}$.

the high-field measurements which show that $K$ and $M_{0}$ are not constant in this temperature range (Fig. 8).

Gaunt $^{7}$ recently used a thermal activation model to extend the absolute-zero coercive fields of the "microdomain" model of Callen et al. ${ }^{8}$ to finite temperatures. Using the recently obtained values $K=1 \times 10^{8}$ $\mathrm{erg} / \mathrm{cm}^{3}$ and $M_{0}=1900 \mathrm{emu} / \mathrm{cm}^{3},{ }^{9}$ the theory ${ }^{7}$ predicts for $\mathrm{Tb}_{75} \mathrm{Au}_{25}$ the coercive field versus temperature as shown in Fig. 5; the fit was obtained with $d=1$ and $V=2000 \AA^{3}$, where $d=K / \lambda M_{0}^{2}$ and $V$ is the domain volume. Following a different approach Egami ${ }^{10}$ explained the temperature dependence of the coercive field assuming a thermal activation of domain walls over energy barriers in highly anisotropic materials. Assuming the absolute-zero coercivity to be $H_{0}=30 \mathrm{kOe}$ and $\langle S\rangle^{2} \mathrm{~g} / n=4.10$ ( $n$ is the domain-wall width in atom spacing units) the predicted $H_{c}$ vs $T$ is plotted on Fig. 5. Both theories explained reasonably well the variation of coercive fields with temperature at liquid-helium temperatures.

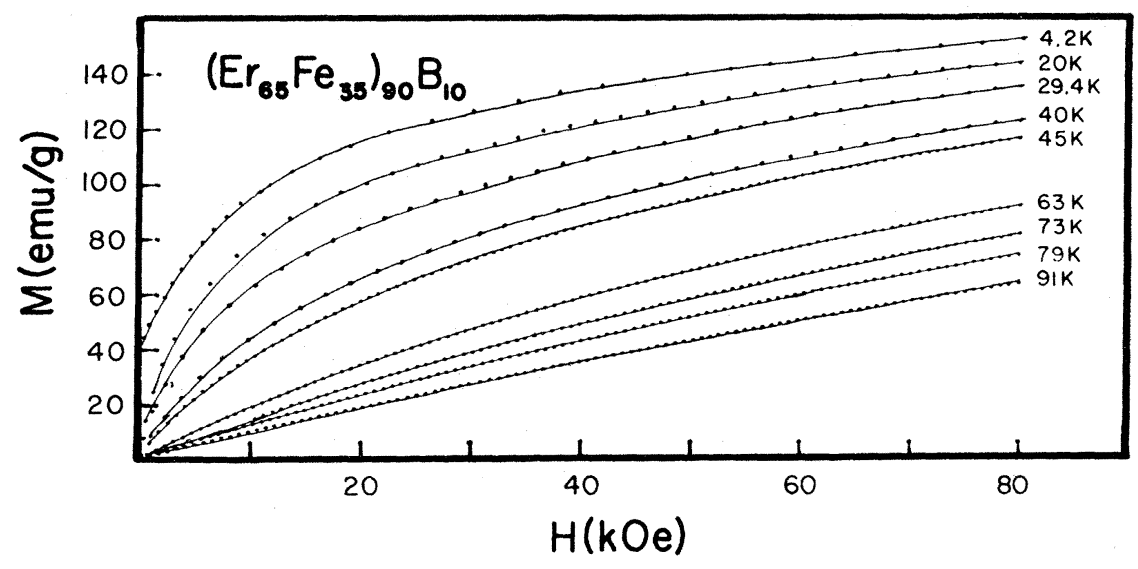

FIG. 8. Magnetization curves for $\left(\mathrm{Er}_{65} \mathrm{Fe}_{35}\right)_{90} \mathrm{~B}_{10}$. 
In the $\mathrm{Tb}$ glass the coercivity at $1.3 \mathrm{~K}$ is found to be lower than the value at $4.2 \mathrm{~K}$. However this may be an artifact since the hysteresis loop at this temperature showed several magnetization jumps which were not symmetrical, thus causing difficulties in determining $H_{c}$. An explanation of the magnetization jumps was given elsewhere. ${ }^{9}$ After the jump the magnetization in a constant field does not vary with time (Fig. 6). This is because most of the energy barriers have been overcome. Higher fields are required to overcome more barriers and cause the magnetization to follow the usual In (time) dependence.

Further analysis of the magnetic aftereffect measurements is not possible without considering a model. If one assumes that the magnetization reversal takes place by domain-wall motion and that the interaction of the domain wall with the inhomogeneities is represented by the interaction energy $V(x)$ then the total energy $E_{t}$ in a field $H$ can be written

$$
E_{t}=V(x)-2 H M A x,
$$

where $M$ is the magnetization per unit volume, $A$ is the area of the domain wall associated with the inhomogeneity, and $x$ is the domain-wall displacement. The values $\pm x_{0}$ of the minimum and maximum energy $E_{t}$ can be found by minimizing Eq. (7).

$$
\frac{\partial E_{t}}{\partial x}=\frac{\partial V}{\partial x}-2 H M A=0 \text {. }
$$

If the interaction energy $V(x)$ is an odd function of position $x$ plus a constant, then the energy barrier is given by, ${ }^{11}$

$$
E=E_{t}\left(+x_{0}\right)-E_{l}\left(-x_{0}\right)=2 E_{t}\left(x_{0}\right),
$$

or

$$
E=2 V\left(x_{0}\right)-4 H M A x_{0},
$$

and

$$
\left(\frac{\partial E}{\partial H}\right)=-4 M A x_{0} .
$$

But $2 A x_{0}$ is the activation volume $V$ where $2 x_{0}$ is the distance the domain wall jumped due to thermal activation of an area $A$ of the wall.

At $4.2 \mathrm{~K}$ the activation volume $V$ is found to be 560 and $2000 \AA^{3}$ for the $\mathrm{Tb}\left(M=2460 \mathrm{emu} / \mathrm{cm}^{3}\right)$ and $\operatorname{Er}\left(M=1370 \mathrm{emu} / \mathrm{cm}^{3}\right)$ glass, respectively. The value of $V$ for the $\mathrm{Tb}$ glass is comparable with the value determined from the coercive field measurements using Gaunt's model ${ }^{7}$ and with the value reported by $\mathrm{Rhyne}$ et al. ${ }^{12}$ for $\mathrm{TbFe}_{2}$, indicating a range of magnetization reversal of a few atomic distances.

\section{CONCLUSION}

The large coercive fields which developed at low temperatures in the non- $S$-state rare-earth amorphous alloys were discussed in terms of recently proposed models of local random anistropy. Strong magnetic aftereffects appear at liquid-helium temperatures suggesting that the temperature dependence of the coercive field is due to thermal activation processes. Magnetization reversal is found to occur in domains of a few atomic distances. The disappearance of hysteresis and magnetic aftereffects well below the ordering temperature indicates the absence of domains above this temperature. However a more precise knowledge of the true magnetic structure is required for a model which can explain the large coercive fields and be consistent with the magnetic viscosity measurements.

\section{ACKNOWLEDGMENTS}

We are grateful to S. G. Cornelison and W. L. Burmester for useful discussions and help with the measurements. We are indebted to the National Science Foundation under Grant No. DMR-7810781 for support of this research.
${ }^{1}$ R. Street and J. C. Wooley, Proc. Phys. Soc. London Ser. A 62, 562 (1949); В $\underline{69}, 1189$ (1956).

2P. Gaunt, Philos. Mag. 34, 775 (1976).

${ }^{3}$ B. Boucher and B. Barbara, J. Phys. F 9, 151 (1979).

${ }^{4}$ D. J. Sellmyer, G. Hadjipanayis, and S. G. Cornelison, J. Non-Cryst. Solids $\underline{40}, 437$ (1980).

5J. A. Gerber, S. G. Cornelison, W. L. Burmester, and D. J. Sellmyer, J. Appl. Phys. 50, 1608 (1979).

${ }^{6}$ C. P. Bean and J. D. Livingston, J. Appl. Phys. 30, 2105 (1959).
${ }^{7}$ P. Gaunt, Phys. Rev. B 19, 521 (1979)

${ }^{8}$ B. Callen, Y. J. Liu, and J. R. Cullen, Phys. Rev. B 16,263 (1977).

${ }^{9}$ G. Hadjipanayis, D. J. Sellmyer, and B. Brandt, Phys. Rev. B 23, 3349 (1981) (preceding paper).

${ }^{10}$ T. Egami, Phys. Status Solidi (a) 20, 157 (1973).

11P. Gaunt and G. J. Roy, Philos. Mag. 34, 781 (1976).

12J. J. Rhyne, J. H. Schelleng, and N. C. Koon, Phys. Rev. B $\underline{10}, 4672$ (1974). 